\title{
Processos de leitura em escolares com Transtorno de Deficit de Atenção/Hiperatividade
}

\author{
Reading processes in students with Attention Deficit Hyperactivity Disorder
}

\author{
Adriana Marques de Oliveira ${ }^{[a]}$, Monique Herrera Cardoso ${ }^{[b]}$, Niura Aparecida de Moura Ribeiro Padula ${ }^{[c]}$, \\ Maria Dalva Lourencetti ${ }^{[d]}$ Lara Cristina Antunes dos Santos ${ }^{[e]}$, Simone Aparecida Capellini ${ }^{[[]}$
}

[a] Fonoaudióloga do Laboratório de Investigação dos Desvios da Aprendizagem (LIDA) do Departamento de Fonoaudiologia da Faculdade de Filosofia e Ciências da Universidade Estadual Paulista Júlio de Mesquita Filho (Unesp), doutoranda do Programa de Pós-Graduação em Educação da Faculdade de Filosofia e Ciências da Universidade Estadual Paulista Júlio de Mesquita Filho (Unesp), Marília, SP - Brasil, e-mail: adrimaroli@yahoo.com.br

${ }^{[b]}$ Fonoaudióloga do Laboratório de Investigação dos Desvios da Aprendizagem (LIDA) do Departamento de Fonoaudiologia da Faculdade de Filosofia e Ciências da Universidade Estadual Paulista Júlio de Mesquita Filho (Unesp), doutoranda do Programa de PósGraduação em Educação da Faculdade de Filosofia e Ciências da Universidade Estadual Paulista Júlio de Mesquita Filho (Unesp), Marília, SP - Brasil, e-mail:moniquehc@gmail.com

${ }^{[c]}$ Neurologista Infantil, doutora em Ciências Médicas pela Universidade Estadual de Campinas (Unicamp), docente do Departamento de Neurologia e Psiquiatria da Faculdade de Medicina da Universidade Estadual Paulista Júlio de Mesquita Filho (Unesp), Botucatu, SP - Brasil, e-mail: niurap@fmb.unesp.br

\section{Resumo}

Este estudo objetiva caracterizar e comparar o desempenho dos escolares com Transtorno de Deficit de Atenção e Hiperatividade (TDAH) com escolares com bom desempenho acadêmico nos processos de leitura. Participaram deste estudo 40 escolares na faixa etária de 8 anos e 2 meses a 10 anos e 11 meses de idade, de ambos os gêneros, de 1a a 4⿳亠丷a série do Ensino Fundamental da cidade de Marília (SP), divididos em: GI, composto por 20 escolares com diagnóstico interdisciplinar de TDAH; GII, 20 escolares com bom desempenho acadêmico, pareados segundo gênero, faixa etária e escolaridade com o GI. Os escolares foram submetidos à aplicação da adaptação brasileira da Avaliação dos Processos de Leitura - PROLEC, composta por quatro blocos: identificação de letras, processos léxicos, sintáticos e semânticos. Foi encontrada diferença estatisticamente significativa entre GI e GII nas provas do PROLEC, com exceção das provas igual-diferente e decisão lexical, indicando que os escolares do GI apresentaram desempenho inferior quando comparados aos escolares do GII. Quanto à classificação dos resultados do PROLEC, observa-se diferença estatisticamente significativa nos processos léxico, sintático, semântico e na prova som e letra, com exceção da prova igual-diferente do processo de identificação de letras. Os achados sugerem que o grupo de escolares com TDAH apresenta desempenho inferior em relação ao grupo sem dificuldades, o que indica que o fator atencional compromete o funcionamento das funções cognitivas, prejudicando a aquisição das habilidades necessárias para o aprendizado da leitura.

Palavras-chave: Avaliação. Leitura. Transtorno de Deficit de Atenção e Hiperatividade.

\section{Abstract}

This study aimed to characterize and compare the performance of students with Attention Deficit Hyperactivity Disorder and students with good academic performance on the reading assessment processes. Forty students ranging from 8 years and 2 months old to 10 years and

1 Trabalho realizado no Laboratório de Investigação dos Desvios da Aprendizagem do Departamento de Fonoaudiologia da Faculdade de Filosofia e Ciências da Universidade Estadual Paulista (FFC/Unesp - Marília, SP, Brasil). 
[d] Neuropsicóloga do Ambulatório de Desvios da Aprendizagem do Hospital das Clínicas da Faculdade de Medicina da Universidade Estadual Paulista Júlio de Mesquita Filho (Unesp), mestre em Saúde Coletiva pela Universidade do Sagrado Coração (USC), Bauru, SP - Brasil, e-mail: dalvalourenceti@ yahoo.com.br

${ }^{[e]}$ Neurologista Infantil, responsável pelo Ambulatório de Desvios da Aprendizagem do Hospital das Clínicas da Faculdade de Medicina da Universidade Estadual Paulista Júlio de Mesquita Filho (Unesp), Botucatu, SP - Brasil, e-mail: lara_ neuropediatria@msn.com

[f] Fonoaudióloga, docente do Departamento de Fonoaudiologia e do Programa de Pós-Graduação em Educação e em Fonoaudiologia da Faculdade de Filosofia e Ciências da Universidade Estadual Paulista Júlio de Mesquita Filho (Unesp), Marília, SP - Brasil, e-mail: sacap@uol.com.br
11 months old, from both genders, from 1st to 4th grades of elementary level, participated in this study, distributed in two groups: GI, with 20 students with interdisciplinary diagnosis of Attention Deficit Hyperactivity Disorder (ADHD), and GII, 20 students with good academic performance, paired with GI according to gender, age and school level. The students were assessed through the Brazilian adaptation of the Assessment Process of Reading - PROLEC, composed of four blocks: identification of letters, lexical, syntactic and semantic processes. Statistically significant difference occurred between GI and GII in the PROLEC tests, with the exception of the same-different and lexical decision tests, indicating that the students from GI presented inferior performance when compared with students from GII. Regarding the classification of the results of PROLEC, there was a statistically significant difference in the lexical, syntactic and semantic processes and the letter and sound test, with the exception of same-different tests of identification of letters process. The findings suggests that the group of students with ADHD shows inferior performance compared to the group without disabilities, highlighting that the attention factor affects the cognitive functions impairing the acquisition of skills necessary for reading.

Keywords: Assessment. Reading. Attention Deficit Hyperactivity Disorder.

Recebido: $14 / 03 / 2011$

Received: 03/14/2011

Aprovado: $17 / 03 / 2011$

Approved: 03/17/2011

\section{Introdução}

0 Transtorno de Deficit de Atenção e Hiperatividade (TDAH) é o distúrbio neuropsiquiátrico mais comum da infância e está incluído entre as doenças crônicas mais prevalentes entre escolares, podendo persistir até a idade adulta (American Academy of Pediatrics, [AAP], 2000; Barkley, Fischer, Smallish, \& Fletcher, 2002; Faraone, 2003; Mattos et al., 2006). São características do quadro: a desatenção, a hiperatividade e a impulsividade, que podem variar em maior ou menor grau (American Psychiatric Association [APA], 2000; Graeff \& Vaz, 2006; Rohde et al., 1999), frequentemente associadas a comprometimentos em atividades cotidianas, vida acadêmica e relações sociais (Kessler et al., 2005; Lima \& Albuquerque, 2003; Pastura, Mattos, \& Araújo, 2005).

O TDAH possui predisposição hereditária, podendo ser agravado por fatores ambientais. A prevalência varia entre $5 \%$ a $15 \%$ das crianças em idade escolar e a incidência é três vezes maior no sexo masculino. Estudos epidemiológicos mais rigorosos definiram taxas de $4 \%$ a $12 \%$ da população geral de crianças de 6 a 12 anos de idade (Moraes, Ciasca, \& Ribeiro, 2006; National Institute of Health, [Nih],
1998; Pastura et al., 2005; Vasconcelos et al., 2003; Vera, Conde, Wajnsztejn \& Nemr, 2006).

Os escolares com TDAH apresentam alterações na função executiva, que abrange todos os processos responsáveis por planejar, focalizar, guiar, direcionar e integrar as funções cognitivas, dentre eles o estado de alerta, atenção sustenta e seletiva (Capovilla, Assef \& Cozza, 2007; Capovilla, A., Cozza, Capovilla, F. \& Macedo, 2005; Grevet, Abreu \& Shansis, 2003; Hurks et al., 2004; Saboya, Saraiva, Palmini, Lima \& Coutinho, 2007).

Em virtude de tais alterações, esses escolares apresentam dificuldade quanto ao aprendizado das habilidades metalinguísticas e aos aspectos fonológicos da linguagem, comprometendo aquisições posteriores, como a aquisição da leitura e da escrita, o que resulta em posteriores dificuldades de aprendizagem (Gallardo-Paúls, Martinéz \& Campos, 2010; Ygual-Fernández, Miranda-Casas \& CerveraMérida, 2000).

0 ato de aprender envolve, em sua dinâmica, a atenção, a memória, o interesse e a inteligência, tornando-se uma ação complexa que pode ou não determinar a apreensão de fatos, conceitos, nomes, conteúdos do currículo escolar e, principalmente, a decodificação de códigos linguísticos (leitura) ou 
aquisição da representação gráfica (escrita) (Bessa, 2006; Pina et al., 2010).

Para ler, a criança deve adquirir certo número de habilidades cognitivas e perceptivo-linguísticas, que incluem atenção dirigida às marcas impressas $\mathrm{e}$ controle dos movimentos de olho pela página, habilidade de focalizar a atenção, a concentração e o seguimento de instruções; habilidade para entender e interpretar a língua falada no cotidiano; memória auditiva e ordenação; memória visual e ordenação; habilidade no processamento das palavras; análise estrutural e contextual da língua; síntese lógica e interpretação da língua; desenvolvimento e expansão do vocabulário; e fluência na leitura (Aaron, Joshi, \& Quatroche, 2008; Ciasca, Capellini, \& Tonelloto, 2003; Flanagan, Ortiz, Alfonso \& Mascolo, 2002; Guidetti \&Martinelli, 2007; Speece \& Ritchey, 2005; Tonelotto \& Gonçalves, 2002).

Este estudo visou caracterizar e comparar o desempenho dos escolares com TDAH com escolares com bom desempenho acadêmico nos processos de leitura.

\section{Método}

Este estudo foi realizado depois da aprovação do Comitê de Ética em Pesquisa da Faculdade de Filosofia e Ciências da Universidade Estadual Paulista (FFC/Unesp - Marília, SP) sob o protocolo n. 26/2011.

Participaram deste estudo 40 escolares na faixa etária de 8 anos e 2 meses a 10 anos e 11 meses de idade, de ambos os gêneros, de $1^{\underline{a}}$ a $4^{\text {a }}$ série do ensino fundamental, divididos em dois grupos:

- Grupo I (GI): composto por 20 escolares, 14 (70\%) do gênero masculino e 6 (30\%) do gênero feminino, com TDAH do Ambulatório de Neurologia Infantil - Desvios de Aprendizagem, do Hospital das Clínicas da Faculdade de Medicina da Unesp, Botucatu.

- Grupo II (GII): composto por 20 escolares, 11 (55\%) do gênero masculino e 9 (45\%) do gênero feminino, com bom desempenho acadêmico, indicados por professores de escola pública do município de Marília (SP) com base no desempenho satisfatório nas provas de Língua Portuguesa e Matemática em dois primeiros bimestres consecutivos, pareados segundo gênero, faixa etária e escolaridade com o GI.

O diagnóstico de TDAH foi realizado por equipe interdisciplinar do Ambulatório de Neurologia Infantil - Desvios da Aprendizagem, do Hospital das Clínicas da Faculdade de Medicina (HC/FM/ Unesp) de Botucatu, incluindo avaliação fonoaudiológica, neurológica, neuropsicológica e seguindo critérios propostos pelo DSM-IV (American Psychiatric Association [APA], 2000). A classificação do nível socioeconômico foi realizada com base no estudo estatístico do Índice de Desenvolvimento Socioeconômico (IDESE) (2003), garantindo, dessa maneira, a homogeneidade da amostra do ponto de vista socioeconômico.

Neste estudo, foram realizados os seguintes procedimentos:

a) Termo de consentimento pós-informado: conforme resolução do Conselho Nacional de Saúde CNS n. 196/96, antes do início das avaliações, os pais ou responsáveis dos pacientes selecionados assinaram o termo de consentimento pós-informado para a autorização da realização do estudo.

b) Avaliação dos processos de leitura: os escolares foram submetidos à aplicação da adaptação brasileira da Avaliação dos Processos de Leitura - PROLEC (Capellini, Oliveira \& Cuetos, 2010). Essa avaliação é composta por quatro blocos distribuídos para a avaliação de quatro processos de leitura, como descritos a seguir:

- 1ํo Processo: identificação de letras; composto por duas provas destinadas a medir a capacidade dos escolares para identificar as letras e seus respectivos sons. A prova de identificação de som e letras visa verificar a capacidade de o escolar nomear as letras e o som que as representa. A prova de igual e diferente no que se refere a palavras e pseudopalavras visa verificar a capacidade de o escolar identificar, discriminar e reconhecer palavras reais e inventadas como sendo igual/diferente.

- 2o Processo: processos léxicos; composto por quatro provas com a finalidade de comprovar o funcionamento das duas rotas de reconhecimento de palavras e seus subprocessos. Na prova de decisão lexical, o escolar deve 
reconhecer apenas palavras reais em uma lista de palavras reais e inventadas independentemente de ser capaz de lê-las ou não. Nas provas de leitura de palavras, leitura de pseudopalavras e leitura de palavras e pseudopalavras, o objetivo é comparar o desenvolvimento das rotas de reconhecimento de palavras, e o escolar deve realizar a leitura de palavras reais e palavras inventadas, sendo que na primeira prova foi medida a capacidade de o escolar ler palavras reais e, na segunda, a capacidade de ler palavras inventadas de diferentes complexidades silábicas, divididas em CCV, VC, CVC, CVV, CCVC e CVVC. $\mathrm{Na}$ terceira prova, o objetivo é analisar o grau de desenvolvimento que o escolar alcançou com o uso de rotas fonológica e lexical para leitura. Para isso, foram utilizadas palavras e pseudopalavras pertencentes a seis categorias: palavras de alta frequência curtas, palavras de alta frequência longas, palavras de baixa frequência curtas, palavras de baixa frequência longas, pseudopalavras curtas e pseudopalavras longas.

- 3 Processo: processos sintáticos; composto por duas provas. Na prova de estruturas gramaticais, verifica-se a capacidade de o escolar processar diferentes tipos de estruturas gramaticais e comprovar a dificuldade que se pode produzir ao utilizar diferentes estruturas sintáticas, a saber: voz ativa, voz passiva e complemento focado. Na prova de sinais de pontuação, observa-se a capacidade de o escolar utilizar sinais de pontuação em um pequeno texto.

- 4o Processo: processos semânticos; composto por duas provas. Na prova de Compreensão de orações, o objetivo é avaliar se o escolar é capaz de extrair o significado de orações simples. Na prova de Compreensão de textos, o objetivo é investigar se o escolar é capaz de extrair o significado e integrá-lo aos seus conhecimentos.

Os escolares do GI foram avaliados no Ambulatório de Neurologia Infantil - Desvios da Aprendizagem, do Hospital das Clínicas da Faculdade de Medicina (HC/FM/Unesp), após 30 minutos da administração do medicamento (metilfenidato), uma vez que na ausência da medicação não foi possível realizar a avaliação proposta neste estudo. Foram realizadas duas sessões para a avaliação, com duração de 30 minutos cada. E os escolares do GII foram avaliados em uma sala de aula fornecida pela coordenação pedagógica, em horário predeterminado pelo professor de cada escolar.

Para a realização da análise estatística, utilizou-se o programa SPSS (Statistical Package for Social Sciences) em sua versão 17.0. Foram aplicados o teste de Mann-Whitney, para comparar o desempenho dos escolares do GI e GII nas provas do PROLEC, e o Teste da Razão de Verossimilhança, para verificar possíveis diferenças entre os grupos quanto à classificação do PROLEC. 0 nível de significância estatística, sinalizado por asterisco $\left({ }^{*}\right)$, foi de $5 \%(0,05)$.

\section{Resultados}

A Tabela 1 apresenta os resultados da descrição e a comparação entre os escolares dos grupos GI e GII nas provas do PROLEC. Com a aplicação do Teste de Mann-Whitney, foi verificada diferença estatisticamente significativa entre GI e GII em quase todas as provas, com exceção das provas igual-diferente (ID), decisão lexical (DL) e no uso da voz passiva (VP), indicando que os escolares do GI apresentaram desempenho inferior quando comparados aos escolares do GII. Observa-se, ainda, maior variabilidade nas respostas do GI em relação ao GII, ou seja, falta homogeneidade nas respostas do GI. As provas que obtiveram maior variabilidade de respostas, tanto no GI como no GII, foram as provas do processo léxico, indicando maior dificuldade dos escolares dos dois grupos em sua realização.

A Tabela 2 apresenta os resultados da comparação entre os escolares do GI e GII quanto à classificação das provas do PROLEC. Com a aplicação do Teste da Razão de Verossimilhança, observou-se diferença estatisticamente significativa em quase todas as provas, com exceção da prova igual-diferente (ID) do processo de identificação de letras, indicando diferença na classificação das provas do PROLEC entre o GI e o GII.

Nota-se, ainda, que a maior parte dos escolares do GII foi classificada com desempenho normal (N) em todas as provas, enquanto que a maioria dos 
Tabela 1 - Comparacãa do desempenho dos escolares do Gl e GIl quanto ao desempenho nas provas do PROLEC

\begin{tabular}{|c|c|c|c|c|c|c|c|c|c|c|}
\hline $\begin{array}{l}\text { Processos } \\
\text { de Leitura }\end{array}$ & Variável & Grupo & Média & $\begin{array}{l}\text { Desvio } \\
\text { padrão }\end{array}$ & Mínimo & Máximo & $\begin{array}{l}\text { Percen- } \\
\text { til } 25\end{array}$ & Mediana & $\begin{array}{c}\text { Percentil } \\
75\end{array}$ & Valor de $p$ \\
\hline \multirow{6}{*}{$\begin{array}{l}\text { Identificação } \\
\text { de Letras }\end{array}$} & \multirow{2}{*}{ SL } & GI & 18,00 & 3,45 & 9,00 & 20,00 & 17,50 & 19,50 & 20,00 & \multirow{2}{*}{$0,001^{*}$} \\
\hline & & GII & 19,95 & 0,22 & 19,00 & 20,00 & 20,00 & 20,00 & 20,00 & \\
\hline & \multirow{2}{*}{ ID } & GI & 18,25 & 4,46 & 0,00 & 20,00 & 19,00 & 20,00 & 20,00 & \multirow{2}{*}{0,425} \\
\hline & & GII & 19,60 & 0,50 & 19,00 & 20,00 & 19,00 & 20,00 & 20,00 & \\
\hline & \multirow{2}{*}{ DL } & GI & 21,50 & 10,22 & 0,00 & 30,00 & 17,00 & 26,50 & 28,00 & \multirow{2}{*}{0,055} \\
\hline & & GII & 27,70 & 1,69 & 25,00 & 30,00 & 26,25 & 27,00 & 29,00 & \\
\hline \multirow{10}{*}{$\begin{array}{l}\text { Processo } \\
\text { Léxico }\end{array}$} & \multirow{2}{*}{ LP } & GI & 22,00 & 10,65 & 0,00 & 30,00 & 17,00 & 27,00 & 30,00 & \multirow{2}{*}{$0,009^{*}$} \\
\hline & & GII & 29,25 & 1,21 & 26,00 & 30,00 & 28,25 & 30,00 & 30,00 & \\
\hline & \multirow{2}{*}{ LPP } & GI & 19,75 & 10,18 & 0,00 & 29,00 & 12,50 & 24,00 & 27,75 & \multirow{2}{*}{$0,008^{*}$} \\
\hline & & GII & 27,20 & 2,26 & 22,00 & 30,00 & 25,25 & 28,00 & 29,00 & \\
\hline & \multirow{2}{*}{ LPF } & GI & 15,25 & 7,42 & 0,00 & 20,00 & 10,50 & 19,00 & 20,00 & \multirow{2}{*}{$<0,001^{*}$} \\
\hline & & GII & 19,90 & 0,31 & 19,00 & 20,00 & 20,00 & 20,00 & 20,00 & \\
\hline & \multirow{2}{*}{ LPNF } & GI & 14,50 & 6,76 & 0,00 & 20,00 & 12,25 & 18,00 & 18,75 & \multirow{2}{*}{$0,006^{*}$} \\
\hline & & GII & 18,75 & 1,45 & 15,00 & 20,00 & 18,00 & 19,00 & 20,00 & \\
\hline & \multirow{2}{*}{ LNP } & GI & 13,25 & 6,67 & 0,00 & 20,00 & 9,00 & 15,50 & 18,00 & \multirow{2}{*}{$0,006^{*}$} \\
\hline & & GII & 17,80 & 2,42 & 10,00 & 20,00 & 17,25 & 18,00 & 20,00 & \\
\hline \multirow{10}{*}{$\begin{array}{l}\text { Processo } \\
\text { Sintático }\end{array}$} & \multirow{2}{*}{ EG } & GI & 8,20 & 4,81 & 0,00 & 14,00 & 5,00 & 10,00 & 11,75 & \multirow{2}{*}{$0,004^{*}$} \\
\hline & & GII & 12,05 & 1,47 & 10,00 & 15,00 & 11,00 & 12,00 & 13,00 & \\
\hline & \multirow{2}{*}{ VA } & GI & 3,20 & 1,85 & 0,00 & 5,00 & 2,25 & 4,00 & 5,00 & \multirow{2}{*}{$0,013^{*}$} \\
\hline & & GII & 4,50 & 0,61 & 3,00 & 5,00 & 4,00 & 5,00 & 5,00 & \\
\hline & \multirow{2}{*}{ VP } & GI & 3,05 & 1,99 & 0,00 & 5,00 & 0,50 & 4,00 & 5,00 & \multirow{2}{*}{0,172} \\
\hline & & GII & 4,10 & 0,79 & 3,00 & 5,00 & 3,25 & 4,00 & 5,00 & \\
\hline & \multirow{2}{*}{$\mathrm{CF}$} & GI & 1,85 & 1,35 & 0,00 & 4,00 & 0,25 & 2,00 & 3,00 & \multirow{2}{*}{$0,003^{*}$} \\
\hline & & GII & 3,20 & 1,01 & 2,00 & 5,00 & 2,00 & 3,00 & 4,00 & \\
\hline & \multirow{2}{*}{ SP } & GI & 5,95 & 4,20 & 0,00 & 10,00 & 0,00 & 8,00 & 9,00 & \\
\hline & & GII & 13,15 & 1,60 & 10,00 & 16,00 & 12,00 & 13,00 & 14,00 & - \\
\hline & $C O$ & GI & 7,45 & 4,43 & 0,00 & 12,00 & 3,50 & 9,50 & 11,00 & ○ $0 \Omega 1 *$ \\
\hline & & GII & 11,85 & 0,37 & 11,00 & 12,00 & 12,00 & 12,00 & 12,00 & $-0,001$ \\
\hline Semântico & & GI & 7,30 & 5,07 & 0,00 & 13,00 & 0,50 & 9,50 & 11,75 & \\
\hline & - & GII & 13,55 & 2,11 & 9,00 & 16,00 & 13,00 & 14,00 & 15,00 & \\
\hline
\end{tabular}

Legenda: SL = nome do som e letra; ID = identificação de igual e diferente; DL = decisão lexical; LP = leitura de palavras; LPP = leitura de pseudopalavras; LPF = leitura de palavras frequentes; LPNF = leitura de palavras não frequentes; LMP = leitura de não palavras; $\mathrm{EG}=$ estrutura gramatical; $\mathrm{VA}=$ voz ativa; $\mathrm{VP}=$ voz passiva; $\mathrm{CF}=$ complemento focado; $\mathrm{SP}=$ sinais de pontuação; $\mathrm{CO}=$ compreensão de oração; $\mathrm{CT}=$ compreensão de texto; ${ }^{*}=$ estatisticamente significativa.

Fonte: Dados da pesquisa.

Nota: Teste de Mann-Whitney, com nível de significância de 5\% (0,05). 
Tabela 2 - Comparação da classificação normal (N), dificuldade pequena (D) e dificuldade grande (DD) do PROLEC dos escolares do GI e GII

\begin{tabular}{lcccccccc}
\hline \begin{tabular}{l} 
Processos de \\
\multicolumn{1}{c}{ Leitura }
\end{tabular} & Classificação & GI & GII & Valor de p & Classificação & GI & GII & Valor de p \\
\hline \multirow{2}{*}{$\begin{array}{l}\text { Identificação de } \\
\text { Letras }\end{array}$} & S.L. N & 14 & 20 & & ID.N & 16 & 20 & \\
& S.L.D. & 1 & 0 & $0,029^{*}$ & ID.D. & 0 & 0 & 0,108 \\
& S.L.DD. & 5 & 0 & & ID.DD. & 4 & 0 & \\
& DL.N. & 12 & 18 & & LPF.N & 13 & 20 & \\
& DL.D. & 2 & 2 & $0,027^{*}$ & LPF.D & 0 & 0 & $0,014^{*}$ \\
Processo & DL.DD. & 6 & 0 & & LPF.DD & 7 & 0 & \\
Léxico & LP.N & 10 & 19 & & LPNF.N & 12 & 19 & \\
& LP.D. & 2 & 0 & $0,006^{*}$ & LPNF.D & 1 & 0 & $0,029^{*}$ \\
& LP.DD & 8 & 1 & & LPNF.DD & 7 & 1 & \\
& LPP.N & 9 & 19 & & LNP.N & 7 & 16 & \\
& LPP.D & 1 & 0 & $0,003^{*}$ & LNP.D & 4 & 2 & $0,013^{*}$ \\
Processo & LPP.DD & 10 & 1 & & LNP.DD & 9 & 2 & \\
Sintático & EG.N & 11 & 20 & & SP.N & 13 & 20 & \\
& EG.D & 2 & 0 & $0,005^{*}$ & SP.D & 0 & 0 & $0,014^{*}$ \\
Processo & EG.DD & 6 & 0 & & SP.DD & 7 & 0 & \\
Semântico & CO.N & 7 & 20 & & CT.N & 4 & 17 & \\
& CO.D & 2 & 0 & $<0,001^{*}$ & CT.D & 5 & 2 & $<0,001^{*}$ \\
\hline
\end{tabular}

Legenda: SL = nome do som e letra; ID = identificação de igual e diferente; DL = decisão lexical; LP: leitura de palavras; LPP = leitura de pseudopalavras; LPF = leitura de palavras frequentes; LPNF = leitura de palavras não frequentes; LNP = leitura de não palavras; $\mathrm{EG}$ = estrutura gramatical; $\mathrm{VA}=$ voz ativa; $\mathrm{VP}=$ voz passiva; $\mathrm{CF}$ = complemento focado; $\mathrm{SP}=$ sinais de pontuação; $\mathrm{CO}=$ compreensão de oração; CT = compreensão de texto; ${ }^{*}=$ estatisticamente significativa.

Fonte: Dados da pesquisa.

Nota: Teste da Razão de Verossimilhança, com nível de significância de 5\% (0,05).

escolares do GI apresentou classificação dificuldade grande (DD) nas provas de leitura de pseudopalavras do processo léxico e nas do processo semântico. Nas demais provas, a maioria dos escolares do GI apresentou desempenho normal $(\mathrm{N})$, porém em menor número que o GII.

\section{Discussão}

Neste estudo, foram encontradas diferenças estatisticamente significativas na prova letra-som do processo de identificação de letra, nas provas do processo léxico, com exceção da prova de decisão lexical e nas provas do processo sintático e semântico, evidenciando o desempenho superior dos escolares com bom desempenho acadêmico em relação aos escolares com TDAH.
O desempenho inferior do GI em relação ao GII pode ser justificado pelas dificuldades dos escolares com TDAH nas habilidades de codificação e decodificação simbólica e nas irregularidades na leitura e escrita, prejudicando a percepção desses escolares sobre o sistema da língua e dificultando a compreensão do princípio alfabético, o que comprometerá aquisições posteriores, como a da leitura e escrita, resultando em posteriores dificuldades de aprendizagem (Cunha \& Capellini, 2009; Gindri, Keske-Soares \& Mota, 2007; Pestun, Omote, Barreto \& Matsuo, 2010; Queiroga, Lins \& Pereira, 2006; Ygual-Fernández et al., 2010).

As alterações presentes nos escolares com TDAH encontram-se nas áreas de percepção, atenção, memória, associação e fixação de informações (Pina et al., 2010). Isso ocorre porque uma pessoa com o quadro de TDAH apresenta menor fluxo sanguíneo 
na região frontal do cérebro, responsável pelo comportamento inibitório (freio), o que influencia a capacidade de planejamento, memória seletiva, regula o estado de vigília e filtra os estímulos, dentre outras funções (Barkley, 2002).

Os escolares com TDAH apresentam em sua história acadêmica o fracasso escolar, que é determinado por alterações na entrada da informação (Banaschewski et al., 2006; Capellini, Ferreira, Salgado \& Ciasca, 2007; Van der Leij \& Morfidi, 2006), pois apresentam dificuldade em integrar os mecanismos neurais responsáveis pelo funcionamento do Sistema Nervoso Central (SNC), afetando, assim, a capacidade de captarem um estímulo - o que, em médio e longo prazo, afeta, particularmente, a aquisição de conteúdos (Relvas, 2007).

Quanto à classificação dos resultados do PROLEC, observa-se diferença estatisticamente significativa nos processos léxico, sintático e semântico e na prova de identificação de som e letras do processo de identificação de letras, não apresentando essa diferença na prova igual-diferente desse processo. Os escolares com TDAH obtiveram classificação dificuldade grande nas provas de leitura de pseudopalavras do processo léxico e nas provas do processo semântico; nas demais provas, a maioria dos escolares do GI apresentou desempenho normal, porém em menor número que o GII.

0 desempenho inferior apresentado pelos escolares com TDAH em relação aos escolares sem dificuldade se justifica à medida que a realização de atividades como leitura de palavras isoladas ou texto necessita de um processamento visual refinado, que está diretamente relacionado ao processamento linguístico da leitura (realizada a identificação da palavra mediante o processo de decodificação fonológica) auxiliado pelo processamento auditivo e ortográfico (Cunha \& Capellini, 2010). Essa interação entre processamento visual, linguístico e auditivo exige função executiva atuante que, quando comprometida, altera a aquisição de estratégias de leitura, mais especificamente de estratégias fonológicas para leitura, resultando em falhas na automatização da decodificação fonológica. Tais falhas geralmente impedem o acesso ao significado das palavras e dos textos, comprometendo, portanto, o objetivo final da leitura, ou seja, a compreensão do texto lido (Capellini et al., 2007; Gallardo-Paúls et al., 2010; Lobo \& Lima, 2008; Martinussen \& Tannock, 2006; Miranda-Casas, Fernandéz, Robledo
\& García-Castellar, 2010; Oliveira \& Albuquerque, 2009; Papazian, Alfonso, Luzondo \& Araguez, 2009).

Neste estudo, pode-se verificar que os escolares do GI apresentam maior dificuldade em relação aos processos léxicos, fonológico e semânticos. Isso se deve ao fato de que o processo atencional é imprescindível à efetivação da aprendizagem, pois a atenção representa a condição inicial e decisiva para as funções cognitivas em sua totalidade, particularmente para o processo de aprendizagem e memorização (Pina et al., 2010). As crianças que apresentam falhas atencionais ou de processamento da informação terão dificuldade para acionar um processamento visual refinado, o que comprometerá o acesso fonológico exigido para a realização da leitura e da escrita de um sistema alfabético (Banaschewski et al., 2006; Gallardo-Paúls et al., 2010; Messina \& Tiedemann, 2009; Miranda-Casas et al., 2010; Papazian et al., 2009; Van der Leij \& Morfidi, 2006).

\section{Considerações finais}

Os escolares do GII apresentaram desempenho superior nas provas do PROLEC em relação aos escolares do GI. Os escolares do GI apresentaram dificuldades na realização das provas letra-som do processo de identificação de letras, nas provas do processo léxico, com exceção da prova de decisão lexical e nas demais provas do processo sintático e semântico.

A maioria dos escolares do GII foi classificada com desempenho normal em todas as provas, enquanto que a maioria dos escolares do GI apresentou classificação dificuldade grande nas provas de leitura de pseudopalavras do processo léxico e nas provas do processo semântico. Nas demais provas, a maioria dos escolares do GI apresentou desempenho normal, porém em menor número que o GII, evidenciando a necessidade da continuidade de estudos com maior número de casos para melhor diferenciação do perfil dos processos de leitura desses escolares.

Dessa forma, este estudo evidenciou que o fator atencional compromete o funcionamento das funções cognitivas, que quando alteradas, prejudicam a aquisição das habilidades necessárias para a leitura comprometendo o acesso ao significado das palavras e textos, bem como a compreensão do material lido. 
•

\section{Referências}

Aaron, P. G., Joshi, R. M., \& Quatroche, D. (2008). Becoming a professional reading teacher. Baltimore, MD: Paul $\mathrm{H}$. Brookes Publishing Co.

American Academy of Pediatrics, [AAP]. (2000). Clinical practice guideline: Diagnosis and evaluation of the child with attention-deficit/hyperactivity disorder. Pediatrics, 105(5), 1158-1170. doi:10.1542/ peds.105.5.1158.

American Psychiatric Association, [APA]. (2000). Diagnostic and statistical manual of mental disorders: DSM-IV-TR. (4 ${ }^{\text {th }}$. ed.). Washington, DC: American Psychiatric Association.

Banaschewski, T., Ruppert, S., Tannock, R., Albrecht, B., Becker, A., Uebel, H., et al. (2006). Colour perception in ADHD.JournalofChild PsychologyandPsychiatric, 47(6), 568-572. doi:10.1111/j.1469-7610.2005.01540.x.

Barkley, R. A. (2002). Transtorno de deficit de atenção - hiperatividade (TDAH). Porto: Editora Porto.

Barkley, R. A., Fischer, M., Smallish, L., \& Fletcher, K. (2002). The persistence of attention-deficit/hyperactivity disorder into young adulthood as a function of reporting source and definition of disorder. Journal of Abnormal Psychology, 111(2), 279-289. doi:10.1037/0021-843X.111.2.279.

Bessa, V. H. (2006). Teorias da aprendizagem. Curitiba: IESDE.

Capellini, S. A., Ferreira, T. L., Salgado, C. A., \& Ciasca, S. M. (2007). Desempenho de escolares bons leitores, com dislexia e com transtorno do déficit de atenção e hiperatividade em nomeação automática rápida. Revista da Sociedade Brasileira de Fonoaudiologia, 12(2), 114-119. doi:10.1590/S1516-80342007000200008.

Capellini, S. A., Oliveira, A. M., Cuetos, F. (2010). PROLEC: Provas de avaliação dos processos de leitura. São Paulo: Casa do Psicólogo.

Capovilla, A. G. S., Assef, E. C. S., \& Cozza H. F. P. (2007). Avaliação neuropsicológica das funções executivas e relação com desatenção e hiperatividade. Avaliação Psicológica, 6(1), 51-60.

Capovilla, A. G. S., Cozza, H. F. Z., Capovilla, F. C., \& Macedo, E. C. (2005). Funções executivas em crianças e correlação com desatenção e hiperatividade. Temas sobre Desenvolvolvimento, 14(82), 4-14.
Ciasca, S. M., Capellini, S. A., \& Tonelotto, J. M. F. (2003). Distúrbios específicos de aprendizagem. In M. C. Ciasca (Ed.). Distúrbios de aprendizagem: Proposta de avaliação interdisciplinar. (pp. 55-65). São Paulo: Casa do Psicólogo.

Cunha, V. L. O., \& Capellini, S. A. (2009). Desempenho de escolares de $1^{\underline{a}}$ a $4^{\underline{a}}$ série do ensino fundamental nas provas de habilidades metafonológicas e de leitura - PROHMELE. Revista da Sociedade Brasileira de Fonoaudiologia, 14(1), 56-68. doi:10.1590/ S1516-80342009000100011.

Cunha, V. L. O., \& Capellini, S. A. (2010). Análise psicolingüística e cognitivo-linguistica das provas de habilidades metalingüísticas e leitura realizadas em escolares de $2^{\underline{a}}$ a $5^{\underline{a}}$ série. Revista Cefac: Atualização Científica em Fonoaudiologia e Educação, 12(5), 772-783.

Faraone, S. V. (2003). Report from the $4^{\text {th }}$ International Meeting of the Attention Deficit Hyperactivity Disorder Molecular Genetics Network. American Journal of Medical Genetics, 121(1), 55-59. doi:10.1002/ajmg.b.20047.

Flanagan, D. P., Ortiz, S. O., Alfonso, V. C., \& Mascolo, J. T. (2002). The achievement test desk reference (ATDR): Comprehensive assessment in learning disabilities. Boston, CT: Allyn and Bacon.

Gallardo-Paúls, B., Martinéz, M. G., \& Campos, V. M. (2010). Pragmática textual em adolescentes con trastorno por déficit de atención/hiperactividad: Argumentación. Revista Neurología, 50(Supl3), 113-117.

Gindri, G., Keske-Soares, M., \& Mota, H. B. (2007). Memória de trabalho, consciência fonológica e hipótese de escrita. Pró-Fono: Revista de Atualização Científica, 19(1), 313-322. doi:10.1590/ S0104-56872007000300010.

Graeff, R. L., \& Vaz, C. E. (2006). Personalidade de crianças com Transtorno de Déficit de Atenção e Hiperatividade (TDAH) por Meio do Rorschach. Psicologia: Teoria e Pesquisa. 22(3), 269-276. doi:10.1590/S0102-37722006000300003.

Grevet, E. H., Abreu, P. B., \& Shansis, F. (2003). Proposta de uma abordagem psicoeducacional em grupos para pacientes adultos com transtorno de déficit de atenção/hiperatividade. Revista de Psiquiatria do Rio Grande do Sul, 25(3), 446-452. doi:10.1590/ S0101-81082003000300006. 
Guidetti, A. A., \& Martinelli, S. C. (2007). Compreensão em leitura e desempenho em escrita de crianças do ensino fundamental. Revista Psicologia, 8(2), 175-84.

Hurks, P. P. M., Hendriksen, J. G. M., Vles, J. S. H., Kalff, A. C., Feron, F. J. M., Kroes, M., et al. (2004).Verbal fluency over time as a measure of automatic and controlled processing in children with ADHD. Brain and Cognition, 55(3), 535-544. doi:/10.1016/j. bandc.2004.03.003.

Kessler, R. C., Adler, L. A., Barkley, R., Biederman, J., Conners, C. K., Faraone, S. V., et al. (2005). Patterns and predictors of attention-deficit/hyperactivity disorder persistence into adulthood: Results from the National Comorbidity Survey Replication. Journal of the Society of Biological Psychiatry, 57(11), 14421451. doi:10.1016/j.biopsych.2005.04.001.

Lima, C. C., \& Albuquerque, G. (2003). Avaliação de linguagem e co-morbidade com transtornos de linguagem. In L. A. Rohde \& P. Mattos (Ed.). Princípios e práticas em TDAH. (pp. 117-142). Porto Alegre: Artmed. PMid:12654532.

Lobo, P. A. S., \& Lima, L. A. M. (2008). Comparação do desempenho em leitura de palavras de crianças com e sem transtorno de déficit de atenção/hiperatividade. Revista Cefac: Atualização Científica em Fonoaudiologia e Educação, 10(4), 471-483.

Martinussen, R., \& Tannock R. (2006). Working memory impairments in children with attention-deficit hyperactivity disorder with and without comorbid language learning disorders. Journal of Clinical and Experimental Neuropsychology, 28(7), 1073-1094. doi:10.1080/13803390500205700.

Mattos, P., Palmini, A., Salgado, C. A., Segenreich, D., Grevet, E., Oliveira, I. R. et al. (2006). Painel brasileiro de especialistas sobre diagnóstico do transtorno de déficit de atenção/hiperatividade (TDAH) em adultos. Revista de Psiquiatria do Rio Grande do Sul, 28(1),5060. doi:10.1590/S0101-81082006000100007.

Miranda-Casas, A., Fernandéz, M. I., Robledo, P., \& GarcíaCastellar, R. (2010). Comprensión de textos de estudiantes con trastorno por déficit de atención/hiperactividad: ¿Qué papel desempeñan las funciones ejecutivas? Revista Neurología, 50(supl3), 135-142.
Moraes, C., Ciasca, S. M., \& Ribeiro, M. V. M. (2006). Problemas escolares e sua associação com transtorno de déficit de atenção e hiperatividade. In L. E. L. R. Valle (Ed.) Neuropsiquiatria: Infância e adolescência. (pp. 33-40). Poços de Caldas, MG: ABENEPI.

National Institute of Health, [NIH]. (1998). Diagnosis and treatment of attention deficit hyperactivity disorder (ADHD). Nih Consensus Statement, 16(2), 1-37.

Oliveira, C. G., \& Albuquerque, P. B. (2009). Diversidade de resultados no estudo do transtorno de déficit de atenção e hiperatividade. Psicologia: Teoria e Pesquisa, 25(1), 93-102. doi:10.1590/ S0102-37722009000100011.

Papazian, O., Alfonso, I., Luzondo, R. J., \& Araguez, N. (2009). Entrenamiento de la función ejecutiva em preescolares con trastorno por déficit de atención/ hiperactividad combinado: Estudio prospectivo, controlado y aleatorizado. Revista Neurología, 48(supl2), 119-122.

Pastura, G. M. C., Mattos, P., \& Araújo, A. P. Q. C. (2005). Desempenho escolar etranstorno do déficit de atenção e hiperatividade. Revista de Psiquiatria Clínica, 32(6), 324-329. doi:10.1590/S0101-60832005000600003.

Pestun, M. S. V., Omote, L. C. F., Barreto, D. C. M., \& Matsuo, T. (2010). Estimulação da consciência fonológica na educação infantil: Prevenção de dificuldades na escrita. Psicologia Escolar e Educacional, 14(1), 95-104. doi:10.1590/S1413-85572010000100011.

Pina, I. L., Macedo, L. S., Sequeira, M. E. A., Silva, I. L., Cardoso, F., Pinto, F. C., et al. (2010). Avaliação de uma intervenção pedagógica na aprendizagem de crianças com Transtorno de Déficit de Atenção e Hiperatividade - TDAH no âmbito das políticas públicas do Estado do Pará. Ensaio: Avaliação e Políticas Públicas em Educação, 18(66), 65-84. doi:10.1590/ S0104-40362010000100005.

Queiroga, B.,Lins, M. B., \& Pereira, M. A. L. V.(2006).Conhecimento morfossintático e ortografia em crianças do ensino fundamental. Psicologia: Teoria e Pesquisa, 22(1), 95-99. doi:10.1590/S0102-37722006000100012.

Relvas, M. P. (2007). Neurociência e transtornos de aprendizagem. Rio de Janeiro: WAK. 
Rohde, L. A., Biederman, J., Busnello, E. A., Zimmermann, H., Schmitz, M., Martins, S., et al. (1999). ADHD in a school sample of Brazilian adolescents: A study of prevalence, comorbid conditions, and impairments. Journal of the American Academy of Child \& Adolescent Psychiatry, 38(6), 716-722. doi:10.1097/00004583-199906000-00019.

Saboya, E., Saraiva, D., Palmini, A., Lima, P., \& Coutinho, G. (2007). Disfunção executiva como uma medida de funcionalidade em adultos com TDAH. Jornal Brasileiro de Psiquiatria, 56(Supl1), 30-33.

Speece, D. L., \& Ritchey, K. D. (2005) A longitudinal study of the development of oral reading fluency in young children at risk for reading failure. Journal of Learning Disabilities, 38(5), 387-399. doi:10.1177/0 0222194050380050201.

Tonelotto, J. M. F., \& Gonçalves, V. M. G. (2002). Autopercepção de crianças desatentas no ambiente escolar. Estudos de Psicologia, 19(3), 31-41. doi:10.1590/S0103-166X2002000300004.
Van der Leij A., \& Morfidi, E. (2006). Core deficits and variable differences in Dutch poor readers learning English. Journal of Learning Disabilities, 39(1), 74-90. doi:10.1177/00222194060390010701.

Vasconcelos, M. M., Werner, J. J., Malheiros, A. F. A., Lima, D. F. N., Santos, I. S. O., \& Barbosa, J. B. (2003). Prevalência do transtorno de déficit de atenção/hiperatividade numa escola pública primária. Arquivos de Neuropsiquiatria, 61(1), 67-73. doi:10.1590/ S0004-282X2003000100012.

Vera, C. F. D., Conde, G. E. S., Wajnsztejn, R., \& Nemr, K. (2006). Transtornos de aprendizagem de presença de respiração oral em indivíduos com diagnóstico de transtorno de déficit de atenção/hiperatividade (TDAH). Revista CEFAC: Atualização Científica em Fonoaudiologia e Educação, 8(4), 441-455.

Ygual-Fernández, A., Miranda-Casas, A., \& CerveraMérida, J. F. (2000). Dificultades em las dimensiones de forma y contenido del lenguaje em los niños com trastorno por déficit de atención com hiperactividad. Revista Neurologia Clínica, 1, 193-202. 